\title{
Emissão de óxido nitroso nos processos de remoção biológica de nitrogênio de efluentes
}

\section{Nitrous oxide emission in the biological nitrogen removal process}

\author{
Marcelo Bortoli \\ Engenheiro Ambiental. Doutorando em Engenharia Química pela Universidade Federal de Santa Catarina (UFSC) - Florianópolis (SC), Brasil. \\ Airton Kunz \\ Químico Industrial. Pesquisador A na Embrapa Suínos e Aves - Concórdia (SC), Brasil. \\ Hugo Moreira Soares \\ Engenheiro Químico. Professor do Departamento de Engenharia Química e de Alimentos da UFSC - Florianópolis (SC), Brasil.
}

Paulo Belli Filho

Engenheiro Sanitarista. Professor do Departamento de Engenharia Sanitária da UFSC - Florianópolis (SC), Brasil.

Rejane Helena Ribeiro da Costa

Engenheira Civil. Professora do Departamento de Engenharia Sanitária da UFSC - Florianópolis (SC), Brasil.

\section{Resumo}

O óxido nitroso $\left(\mathrm{N}_{2} \mathrm{O}\right)$ é altamente impactante ao meio ambiente por ser um dos três gases mais importantes quando considerado o alto potencial de efeito estufa e a baixa quantidade emitida para a atmosfera. A preocupação com a geração de $\mathrm{N}_{2} \mathrm{O}$ no tratamento de efluentes tem crescido nas duas últimas décadas. Muitos estudos vêm sendo realizados com o objetivo de avaliar as condições de geração e emissão de $\mathrm{N}_{2} \mathrm{O}$ em etapas de remoção de nitrogênio no tratamento, tanto em escala laboratorial quanto em estações de tratamento de efluentes. Essas pesquisas demonstram que, sob certas condições, ambos os processos podem produzir e emitir grandes quantidades de $\mathrm{N}_{2} \mathrm{O}$ para a atmosfera, o que remete à importância de mais investigações para determinar as condições específicas que minimizem a produção e a emissão de $\mathrm{N}_{2} \mathrm{O}$ nesse caso.

Palavras-chave: óxido nitroso; nitrificação; desnitrificação; tratamento de efluentes.

\section{Abstract}

The nitrous oxide $\left(\mathrm{N}_{2} \mathrm{O}\right)$ has a high striking power in environmental. It's one of the three most important greenhouse gases, when considered the greenhouse potential and emissions to the atmosphere. The concern in the two last decades with the $\mathrm{N}_{2} \mathrm{O}$ generation in wastewater treatment has grown. Many studies have been conducted with the objective of evaluate the conditions of $\mathrm{N}_{2} \mathrm{O}$ generation and emission in the nitrification and denitrification process, in biological nitrogen removal of wastewater treatment, both lab scale and wastewater treatment plants (WWTP). These studies show that under certain conditions, both processes can generate and emit large amounts of $\mathrm{N}_{2} \mathrm{O}$ to the atmosphere, what demonstrates the importance of conducting further investigations to determine specific conditions that minimize $\mathrm{N}_{2} \mathrm{O}$ production and emission.

Keywords: nitrous oxide; nitrification; denitrification; wastewater treatment. 


\section{INTRODUÇÃO}

A década de 1950 foi um marco para os estudos relacionados às mudanças climáticas, pois nesse período iniciaram-se efetivamente as medições, com alta confiabilidade, diretamente na atmosfera do dióxido de carbono $\left(\mathrm{CO}_{2}\right)$. Com isso, comprovaram-se as perspectivas da época, de que a ação antropogênica interferia efetivamente no aumento da concentração dos gases de efeito estufa na atmosfera. Contudo, somente na década de 1970 detectaram-se, com considerável abundância, outros dois importantes gases do tipo, presentes na atmosfera e emitidos com significativa relevância: o metano $\left(\mathrm{CH}_{4}\right)$ e o óxido nitroso ( $\mathrm{N}_{2} \mathrm{O}$ ) (IPCC, 2006).

Sabe-se que a concentração de $\mathrm{N}_{2} \mathrm{O}$ aumentou na atmosfera relativamente mais no período entre 1998 e 2005 que entre último ciclo glacial-interglacial (IPCC, 2006).

A emissão de $\mathrm{N}_{2} \mathrm{O}$ tem contribuição significativa por possuir potencial de efeito estufa 300 vezes maior em comparação com o $\mathrm{CO}_{2}$, sendo atualmente o terceiro mais importante gás de efeito estufa presente na atmosfera, atrás somente do $\mathrm{CO}_{2}$ e do $\mathrm{CH}_{4}$. Seu tempo de vida pode ser superior a 120 anos, além de que pode ser destruído na estratosfera, formando NO e contribuindo com a degradação da camada de ozônio (OLIVER et al., 1998; JOHNSTON, 1971).

Outro aspecto que precisa ser observado é que o $\mathrm{N}_{2} \mathrm{O}$ pode ser produzido por atividades humanas, como o uso de fertilizantes no solo e a queima de combustíveis fósseis, além de nos sistemas de tratamento de efluentes (STEs). A agricultura global contribui com entre 65 e $80 \%$ de todo o $\mathrm{N}_{2} \mathrm{O}$ emitido para atmosfera, principalmente proveniente do nitrogênio presente nos fertilizantes aplicados no cultivo do solo. Quase $90 \%$ do $\mathrm{N}_{2} \mathrm{O}$ da atmosfera foram gerados durante a transformação microbiológica da $\mathrm{NH}_{3} \mathrm{em}$ $\mathrm{NO}_{3}^{-}$(IPCC, 2001).

$\mathrm{O} \mathrm{N}_{2} \mathrm{O}$ é abundantemente produzido pela atividade microbiológica. Várias pesquisas citam que é na etapa de remoção de nitrogênio em STEs que acontecem as maiores emissões de $\mathrm{N}_{2} \mathrm{O}$ (TALLEC et al., 2006).Porém, Czepiel et al. (1995), que realizaram diversas medidas da emissão de $\mathrm{N}_{2} \mathrm{O}$ em STEs, observaram que $90 \%$ das emissões são provenientes de lodos ativados, 5\% de caixas de separação de areia e 5\% de tanques de estocagem de lodo. Outros autores afirmam que é na desnitrificação que o $\mathrm{N}_{2} \mathrm{O}$ por ser um intermediário da cadeia respiratória dos microrganismos desnitrificantes (BRETTAR e HOFLE, 1993) e, com isso, a etapa em questão seria, sob certas condições, a de maior emissão de $\mathrm{N}_{2} \mathrm{O}$ (TALLEC et al., 2008).

Contudo, pouco se sabe quanto às reais emissões de $\mathrm{N}_{2} \mathrm{O}$ na remoção biológica de nitrogênio de efluentes, principalmente por ocorrer em condições específicas e muitas vezes em períodos curtos, além da difícil quantificação das emissões de reatores em escala real. Portanto, o assunto é bastante discutido e divergente na literatura.

\section{A GERAÇÃO DE N 20 NOS PROCESSOS BIOLÓGICOS}

O nitrogênio sofre diversas transformações durante vários processos biológicos. Em todos eles, os microrganismos retiram energia das reações de oxirredução das formas nitrogenadas. O ciclo biológico do nitrogênio é complexo e envolve diversas reações metabólicas de diferentes microrganismos.

\section{Nitrificação}

As bactérias oxidadoras de amônia (BOAs) oxidam amônia $\left(\mathrm{NH}_{3}\right)$ a nitrito $\left(\mathrm{NO}_{2}^{-}\right)$, enquanto as oxidadoras de nitrito (BONs) convertem $\mathrm{O} \mathrm{NO}_{2}{ }^{-}$a nitrato $\left(\mathrm{NO}_{3}{ }^{-}\right)$em uma única etapa e ambas, BOAs e BONs, utilizam o $\mathrm{CO}_{2}$ como fonte de carbono, ou seja, são microrganismos autotróficos (YE e THOMAS, 2001).

Nas diferentes etapas da oxidação da $\mathrm{NH}_{3}$ a $\mathrm{NO}_{3}^{-}$, as reações são catalisadas por enzimas específicas. As reações mais complexas ocorrem na primeira etapa, a de nitritação ou oxidação da $\mathrm{NH}_{3}$ a $\mathrm{NO}_{2}{ }^{-}$, na qual aparecem intermediários como hidroxilamina $\left(\mathrm{NH}_{2} \mathrm{OH}\right)$. Duas enzimas que participam dessas reações são as mais importantes: a amônia mono-oxigenase, que age na conversão de $\mathrm{NH}_{3}$ para $\mathrm{NH}_{2} \mathrm{OH}$, e a hidroxilamina oxidoredutase, na conversão de $\mathrm{NH}_{2} \mathrm{OH}$ em $\mathrm{NO}_{2}$ : Existem outras enzimas catalisando reações na região da parede celular das bactérias oxidadoras de amônia, como a nitrito redutase, que age na redução do $\mathrm{NO}_{2}^{-}$a $\mathrm{NO}$ e a óxido nítrico redutase, catalisadora da redução do $\mathrm{NO}$ a $\mathrm{N}_{2} \mathrm{O}$ (KLOTZ e STEIN 2007).

Há bactérias heterotróficas e arqueas metanogênicas também capazes de oxidarem a $\mathrm{NH}_{3}$ a $\mathrm{NH}_{2} \mathrm{OH}$, porém essa rota é muito desfavorável energeticamente (ANDERSON e LEVINE, 1986), ocorre com taxas de 100 a 1.000 vezes mais lentas que a oxidação autotrófica, sob condições de relação DQO/N acima de 10 e baixo oxigênio dissolvido no meio (VAN NIEL et al., 1993).

\section{Desnitrificação}

A desnitrificação faz parte do ciclo do nitrogênio; é a transformação do $\mathrm{NO}_{3}$ a $\mathrm{N}_{2}$ na ausência de $\mathrm{O}_{2}$ (YE e THOMAS, 2001). Cada etapa dessa reação é catabolizada por metaloenzimas específicas. Além disso, já se sabe que pode ser mais de um tipo de redutase por etapa. Em geral, as enzimas necessárias para a desnitrificação somente são produzidas sob, ou próximo a, condições anaeróbias, e se as células em crescimento anaeróbio forem expostas ao $\mathrm{O}_{2}$ essas enzimas são prontamente inibidas. Ainda, as reações são catabólicas, ou seja, regidas por microrganismos heterótrofos, necessitando de matéria orgânica, como fonte de carbono, para a sua síntese celular (SPANNING et al., 2007).

O NO é gerado pela nitrito redutase, mas em baixas concentrações por causa da sua toxicidade. Todavia, este não deixa de ser um intermediário livre da desnitrificação. A óxido nítrico redutase é uma 
enzima presente na membrana celular que participa da redução do $\mathrm{NO}$ a $\mathrm{N}_{2} \mathrm{O}$. A etapa final da desnitrificação é catalisada pela óxido nitroso redutase, outra enzima periplasmática, agindo na redução do óxido nitroso a nitrogênio gasoso (SPANNING et al., 2007).

Sabe-se ainda que existem BOAs capazes de desnitrificar a partir do $\mathrm{NO}_{2}$ - e levando à formação de $\mathrm{N}_{2} \mathrm{O}$, com a $\mathrm{NH}_{3}$ ou hidrogênio como doadores de elétrons. Esse processo também é conhecido como nitrificador desnitrificante (BOCK et al., 1995).

Tanto a desnitrificação aeróbia quanto o nitrificador desnitrificante produzem mais $\mathrm{N}_{2} \mathrm{O}$ relativo ao nitrogênio removido quando comparados à desnitrificação heterotrófica (COLLIVER e STEPHENSON, 2000).

\section{FATORES POTENCIALIZADORES DA EMISSÃO DE $\mathrm{N}_{2} \mathrm{O}$}

Alguns fatores operacionais interferem diretamente na maximização da produção e geração de $\mathrm{N}_{2} \mathrm{O}$ tanto no processo de nitrificação quanto no de desnitrificação (Figura 1), como a relação entre o carbono disponível e o nitrogênio encontrada nos esgotos, que acaba por interferir no processo de desnitrificação (BERNET et al., 1996) ou a concentração de oxigênio dissolvido tanto no processo nitrificante quanto no desnitrificante (TSUNEDA et al., 2005).

\section{Relação entre disponibilidade de carbono e nitrogênio}

A relação entre carbono (C) ou demanda química de oxigênio (DQO) /nitrogênio (C ou DQO/N) é um importante parâmetro de controle de produção de $\mathrm{N}_{2} \mathrm{O}$. Quando a relação DQO/N é menor que 3,5 são observadas mais emissões de $\mathrm{N}_{2} \mathrm{O}$ do que quando a mesma é maior que 3,5. Itokawa et al. (2001) realizaram experimentos com relações DQO/N de 2,4, 3,5 e 5,0 e observaram emissões de 24,4, 59,2 e 0\%, respectivamente, de $\mathrm{N}_{2} \mathrm{O}$ relativo ao nitrogênio removido.
Em outro estudo observou-se que em relações $\mathrm{C} / \mathrm{N}$ entre 1,56 e 2,38 ocorreu redução de todo o $\mathrm{NO}_{3}^{-}$, porém com significativa quantidade de $\mathrm{N}_{2} \mathrm{O}$ presente na fração gasosa. Já com relação $\mathrm{C} / \mathrm{N}$ acima de 18,04, como facilmente encontrada em dejetos animais, observou-se completa desnitrificação, ou seja, sem presença de $\mathrm{N}_{2} \mathrm{O}$ (BERNET et al., 1996).

Contudo, elevadas relações $\mathrm{C} / \mathrm{N}$, acima de dez, em reatores sequenciais ou pré-desnitrificantes, o excesso de carbono pode aumentar a desnitrificação aeróbia e, consequentemente, os níveis de emissão de $\mathrm{N}_{2} \mathrm{O}$ (VAN NIEL et al., 1993).

Em culturas puras, pode-se citar o experimento de Schalk-Otte et al. (2000), que estudaram culturas puras de A. faecalis e observaram que de 32 a 64\% do nitrogênio removido foram emitidos na forma de $\mathrm{N}_{2} \mathrm{O}$ quando submetidas à falta de carbono biodisponível.

\section{Diferentes fontes de carbono}

A natureza da fonte do carbono normalmente especifica sua biodisponibilidade e interfere diretamente na quantidade de $\mathrm{N}_{2} \mathrm{O}$ emitida na desnitrificação (KARGI e PAMUKOGLU, 2003). Outro fator de importância com relação ao carbono biodisponível é que as culturas de microrganismos em reatores utilizados no tratamento de efluentes são de impressionante variabilidade de espécies e gêneros, ocorrendo grande competição pela utilização do carbono biodisponível (RIVETT et al., 2008)

$\mathrm{Na}$ desnitrificação de efluentes, observa-se que os microrganismos têm preferência, com relação à fonte de carbono, pelos ácidos orgânicos voláteis (AOV), que são ácidos de cadeia curta. Dentre eles, três são os principais: ácido acético, propiônico e butírico (ELEFSINIOTIS E WAREHAM, 2007).

Acetato e etanol são comumente utilizados como fonte de carbono em etapas desnitrificantes de tratamento de efluentes a

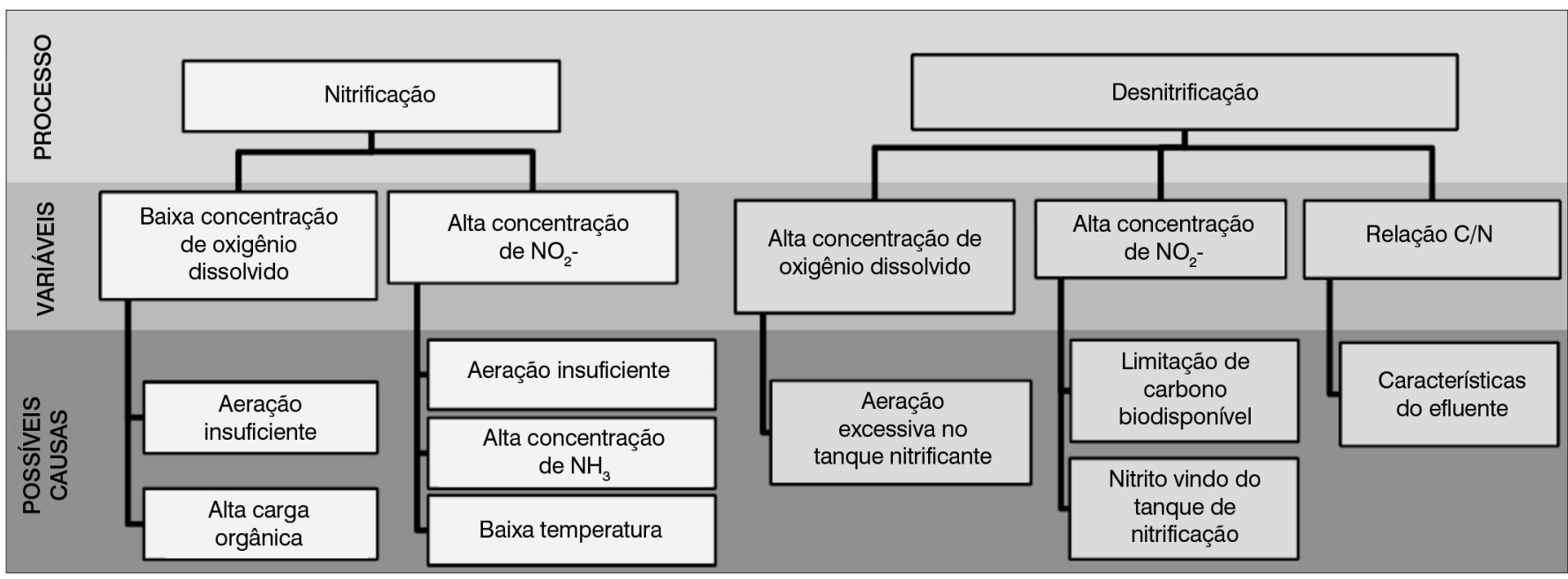

Figura 1 - Variáveis que potencializam a emissão de $\mathrm{N}_{2} \mathrm{O}$ na remoção biológica de nitrogênio de efluentes e possíveis causas de influência sobre as mesmas (adaptado de Kampschreur et al., 2009). 
fim de suplementar o carbono insuficiente presente no efluente, são substratos facilmente utilizados pelos microrganismos desnitrificantes, até mesmo sob condições desfavoráveis com temperaturas abaixo de $10^{\circ} \mathrm{C}$ (MARTIN et al., 2009). No entanto, em se tratando da emissão de $\mathrm{N}_{2} \mathrm{O}$ na etapa desnitrificante, o acetato tem potencial de emissão superior ao metanol em mais de 70 vezes, sob as mesmas condições de operação (ADOUANI et al., 2010).

\section{Concentração de oxigênio dissolvido}

A concentração de oxigênio dissolvido é considerada um parâmetro importante com relação à emissão de $\mathrm{N}_{2} \mathrm{O}$ nos processos nitrificantes, isso porque baixas concentrações de oxigênio dissolvido na etapa nitrificante ocasionam elevadas taxas de produção de $\mathrm{N}_{2} \mathrm{O}$ (KAMPSCHREUR et al., 2008). Sob condições de aeração completa, sem falta de oxigênio, a produção de $\mathrm{N}_{2} \mathrm{O}$ na nitrificação é muito pequena (TALLEC et al., 2006).

Concentrações de oxigênio dissolvido em culturas puras de Nitrossomonas sp. abaixo de $0,5 \mathrm{mg} \cdot \mathrm{L}^{-1}$ apresentaram produção de $\mathrm{N}_{2} \mathrm{O}$ elevadas, entre 3,1 e 9,9\% do nitrogênio removido, enquanto concentrações de oxigênio dissolvido acima de 3,5 mg.L-1 apresentaram produção de 0,26\% de $\mathrm{N}_{2} \mathrm{O}$ (GOREAU et al., 1980).

Em medidas de diversos STEs em escala real, foi observado que com a queda do oxigênio dissolvido ocorreu considerável incremento na emissão de $\mathrm{N}_{2} \mathrm{O}$. Contudo, o aumento súbito da concentração de oxigênio dissolvido também ocasiona aumento na emissão de $\mathrm{N}_{2} \mathrm{O}$ em função das BOAs terem taxa de crescimento maior que as BONs. Com isso ocorre o acúmulo de $\mathrm{NO}_{2}{ }^{-}$e, consequentemente, o aumento da produção de $\mathrm{N}_{2} \mathrm{O}$ (AHN et al., 2010).

$\mathrm{O} \mathrm{N}_{2} \mathrm{O}$ produzido em reatores aeróbios pode ser atribuído basicamente à desnitrificação incompleta realizada pelas BOAs. Na falta de oxigênio, as $\mathrm{BOAs}$ utilizam o $\mathrm{NO}_{2}{ }^{-}$como aceptor final de elétrons na tentativa de manter o oxigênio mínimo necessário para a oxidação da amônia à hidroxilamina (YU et al., 2010).

A vazão de ar nos tanques de nitrificação interfere diretamente na quantidade de $\mathrm{N}_{2} \mathrm{O}$ que acaba por ser emitido para a atmosfera, isso porque com vazões baixas não se mantêm concentrações mínimas necessárias para nitrificação, e com vazões elevadas ocorre o stripping do $\mathrm{N}_{2} \mathrm{O}$ (KAMPSCHREUR et al., 2009).

Quanto à presença de oxigênio dissolvido na etapa desnitrificante, é um importante fator de controle da emissão de $\mathrm{N}_{2} \mathrm{O}$. Efluentes com baixa concentração inicial de oxigênio dissolvido apresentaram total redução do $\mathrm{NO}_{3}{ }^{-}$fornecido à $\mathrm{N}_{2}$. Com concentração próxima a 0,9 mg. $\mathrm{L}^{-1}$ de oxigênio dissolvido, fornecendo como fonte de carbono ácido butírico e ácido glutâmico, observou-se considerável aumento na emissão de $\mathrm{N}_{2} \mathrm{O}$ (MORLEY e BAGGS, 2010).

Já é de notável conhecimento que o oxigênio interfere diretamente na eficiência de desnitrificação, agindo principalmente na enzima óxido nitroso redutase que, por ter maior inibição pelo oxigênio que as demais enzimas envolvidas, faz com que o $\mathrm{N}_{2} \mathrm{O}$ acumule no meio (TSUNEDA et al., 2005).

\section{Concentração de nitrito}

Concentrações elevadas de $\mathrm{NO}_{2}^{-}$também interferem positivamente na emissão de $\mathrm{N}_{2} \mathrm{O}$, tornando $\mathrm{O} \mathrm{NO}_{2}^{-}$um dos parâmetros importantes na emissão de $\mathrm{N}_{2} \mathrm{O}$ na nitrificação em lodos ativados. Esse fato é observado em reatores com predominância de ambos os microrganismos Nitrossomonas europaea (BEAUMONT et al., 2004) e Nitrospira spp. (SHAW et al., 2006).

Elevadas concentrações de $\mathrm{NO}_{2}{ }^{-}$na etapa de nitrificação acima de $300 \mathrm{mg} \cdot \mathrm{L}^{-1}$ acabam por favorecer a desnitrificação pelas BOAs, com redução do $\mathrm{NO}_{2}{ }^{-}$até $\mathrm{N}_{2} \mathrm{O}$. A desnitrificação autotrófica realizada pelas $\mathrm{BOAs}$ emite elevadas quantidades de $\mathrm{N}_{2} \mathrm{O}$ principalmente em função das BOAs não terem a capacidade de produzir a enzima óxido nitroso redutase (YU et al., 2010).

Inicialmente, estudos propunham que a produção de $\mathrm{N}_{2} \mathrm{O}$ pelas $\mathrm{BOAs}$, em condições de elevada concentração de $\mathrm{NO}_{2}{ }^{-}$, estaria associada à imposição de baixas concentrações de oxigênio dissolvido (GRAFF et al., 2010). Porém, recentemente, pesquisas atribuíram tal acontecimento não à falta de oxigênio disponível para as BOAs e sim a uma fase de recuperação das BOAs a concentrações baixas de oxigênio dissolvido (YU et al., 2010).

$\mathrm{Na}$ etapa desnitrificante, vários estudos apontam a interferência positiva na emissão de $\mathrm{N}_{2} \mathrm{O}$ na presença de elevadas concentrações de $\mathrm{NO}_{2}$. Contraditoriamente, estudos citam que a inibição da redução do $\mathrm{N}_{2} \mathrm{O}$ na desnitrificação acontece também com baixas concentrações de $\mathrm{NO}_{2}{ }^{-}$, próximas a $1 \mathrm{mg} \mathrm{N}-\mathrm{NO}_{2}{ }^{-} \cdot \mathrm{L}^{-1}$ (ZHENG et al., 1994). Outros autores citam que a inibição pode acontecer a $5 \mathrm{mg}$ ${\mathrm{N}-\mathrm{NO}_{2}}^{-} \cdot \mathrm{L}^{-1}$ (LEMAIRE et al., 2006) e a $10 \mathrm{mg} \mathrm{N}-\mathrm{NO}_{2} \cdot \mathrm{L}^{-1}$ (HANAKI et al., 1992).

\section{Alterações bruscas no processo}

Alguns autores citam como um dos fatores que maximizam a emissão de $\mathrm{N}_{2} \mathrm{O}$ na remoção biológica de nitrogênio em STEs as mudanças repentinas nas condições ambientais e de operação dos reatores. Basicamente, os microrganismos necessitam de um tempo para se adaptarem às novas condições, e as mudanças afetam as reações do metabolismo, tornando-as incompletas (KAMPSCHREUR et al., 2009).

Alterações de carga de alimentação de amônia afetam diretamente os processos de nitrificação e desnitrificação, maximizando a emissão de $\mathrm{N}_{2} \mathrm{O}$ (BURGESS et al., 2002). Outros autores citam mudanças nas concentrações de $\mathrm{NO}_{2}{ }^{-}$como outra fonte de interferência positiva na emissão de $\mathrm{N}_{2} \mathrm{O}$, além de mudanças nas concentrações de oxigênio dissolvido (KAMPSCHREUR et al., 2008). 
$\mathrm{pH}$

Quanto à influência do pH, Wicht et al. (1996) afirmaram que a inibição da desnitrificação pelo pH está diretamente vinculada à concentração de $\mathrm{NO}_{2}{ }^{-}$e $\mathrm{HNO}_{2}$. Os autores observam elevada emissão de $\mathrm{N}_{2} \mathrm{O}$ na desnitrificação causada pela inibição da enzima óxido nitroso redutase por $\mathrm{HNO}_{2}$ em pH abaixo de 7. Thoern e Soerensson (1996) obtiveram resultado semelhante quando observaram a formação de $\mathrm{N}_{2} \mathrm{O}$ na desnitrificação em pH abaixo de 6,8. Hanaki et al. (1992) impuseram queda do pH de 8,5 para 6,5 e observaram aumento na emissão de $\mathrm{N}_{2} \mathrm{O}$.

Já na nitrificação, Hynes and Knowles (1984) observaram elevada emissão de $\mathrm{N}_{2} \mathrm{O}$ em pH, acima de 8,5, em cultura puras de Nitrossomonas Europae. Segundo Graff et al. (2010), os níveis de produção de $\mathrm{N}_{2} \mathrm{O}$ pelas BOAs em índices de $\mathrm{pH}$ entre 6,3 e 7,7 foram baixos, entre 1 e 2\% do nitrogênio removido.

\section{CONCLUSÕES}

$\mathrm{O} \mathrm{N}_{2} \mathrm{O}$ tem grande destaque no ambiente por ser considerado o terceiro mais importante gás de efeito estufa. É gerado em grandes quantidades nas etapas de remoção de nitrogênio em STEs, sob determinadas condições.

A concentração de oxigênio dissolvido é o parâmetro mais importante na emissão de $\mathrm{N}_{2} \mathrm{O}$, tanto na nitrificação quanto na desnitrificação. Na nitrificação, maximiza a produção de $\mathrm{N}_{2} \mathrm{O}$ em baixas concentrações, e na desnitrificação inibe a expressão da óxido nitroso redutase por parte das bactérias desnitrificantes.

Outro parâmetro importante é a presença de nitrito, que em altas concentrações, tanto na nitrificação quanto na desnitrificação, aumenta a emissão de $\mathrm{N}_{2} \mathrm{O}$. Porém, na nitrificação tal fato está diretamente ligado à concentração de oxigênio dissolvido no meio.

A relação $\mathrm{C} / \mathrm{N}$ (ou $\mathrm{DQO} / \mathrm{N}$ ) nos processos desnitrificantes tem grande participação na formação de $\mathrm{N}_{2} \mathrm{O}$, principalmente quando essa relação $(\mathrm{C} / \mathrm{N})$ é menor que dois. Porém autores citam que a relação $\mathrm{C} / \mathrm{N}$ acima de dez pode favorecer a desnitrificação aeróbia, fator que contribui para o aumento na emissão de $\mathrm{N}_{2} \mathrm{O}$.

Contudo, os estudos apresentam grande variação nas medições de produção e emissão de $\mathrm{N}_{2} \mathrm{O}$ em diferentes condições e processos, demonstrando a necessidade de mais estudos das condições específicas que as favorecem.

\section{Referências}

ADOUANI, N.; LENDORMI, T.; LIMOUSY, L.; SIRE, O. (2010) Effect of the carbon source on $\mathrm{N}_{2} \mathrm{O}$ emissions during biological denitrification. Resources, Conservation and Recycling, v. 54, n. 5, p. 299-302.

AHN, J.H.; KIM, S.; PARK, H.; RAHM, B; PAGILLA, K.; CHANDRAN, K. (2010) $\mathrm{N}_{2} \mathrm{O}$ emissions from activated sludge processes, 2008-2009: results of a national monitoring survey in the United States. Environmental Science \& Technology, v. 44, n. 12, p. 4505-11.

ANDERSON, I.C.; LEVINE, J.S. (1986) Relative rates of nitric oxide and nitrous oxide production by nitrifiers, denitrifiers, and nitrate respirers. Applied and Environmental Microbiology, v. 51, n. 5, p. 938-45

BEAUMONT, H.J.E.; VAN SCHOOTEN, B.; LENS, S.I.; WESTERHOFF, H.V.; VAN SPANNING, R.J.M. (2004) Nitrosomonas europaea expresses a nitric oxide reductase during nitrification. Journal of Bacteriology, $\mathrm{v}$. 186, n. 13, p. 4417-21.

BERNET, N.; DELGENES, N.; MOLETTA, R. (1996) Denitrification by anaerobic sludge in piggery wastewater. Environmental Technology, v. 17, n. 3, p. 293-300.

BOCK, E.; SCHMIDT, I.; STUVEN, R.; ZART, D. (1995) Nitrogen loss caused by denitrifying Nitrosomonas cells using ammonium or hydrogen as electron-donors and nitrite as electron-acceptor. Archives of Microbiology, v. 163, n. 1, p. 16-20.

BURGESS, J.E.; COLLIVER, B.B.; STUETZ, R.M.; STEPHENSON, T. (2002) Dinitrogen oxide production by a mixed culture of nitrifying bacteria during ammonia shock loading and aeration failure.
Journal of Industrial Microbiology and Biotechnology, v. 29, n. 6 , p. 309-13

COLLIVER, B.B.; STEPHENSON, T. (2000) Production of nitrogen oxide and dinitrogen oxide by autotrophic nitrifiers. Biotechnology Advances, $\mathrm{v}$. 18, n. 3, p. 219-32.

CZEPIEL, P.; CRILL, P.; HARRISS, R. (1995) Nitrous oxide emissions from municipal wastewater treatment. Environmental Science \& Technology, v. 29, n. 9, p. 2352-6.

ELEFSINIOTIS, P.; WAREHAM, D.G. (2007) Utilization patterns of volatile fatty acids in the denitrification reaction. Enzyme and Microbial Technology, v. 41, n. 1-2, p. 92-7.

FREITAG, A.; BOCK, E. (1990) Energy conservation in Nitrobacter. FEMS Microbiology Letters, v. 66, n. 1-3, p. 157-62.

GOREAU, T.J.; KAPLAN, W.A.; WOFSY, S.C.; MCELROY, M.B.; VALOIS F.W.; WATSON, S.W. (1980) Production of $\mathrm{NO}_{2}$ - and $\mathrm{N}_{2} \mathrm{O}$ by nitrifying bacteria at reduced concentrations of oxygen. Applied and Environmental Microbiology, v. 40, n. 3, p. 526-32.

HANAKI, K.; HONG, Z; MATSUO, T. (1992) Production of nitrous oxide gas during denitrification of wastewater. Water Science \& Technology, v. 26, n. 5-6, p. 1027-36.

HYNES, R.K.; KNOWLES, R. (1984) Production of nitrous oxide by Nitrosomonas europaea: effects of acetylene, $\mathrm{pH}$, and oxygen. Canadian Journal of Microbiology, v. 30, n. 11, p. 1397-404. 
IPCC AR3. (2001) Climate Change 2001: the scientific basis. Contribution of working group I to the Third Assessment Report of the Intergovernmental Panel on Climate Change. Cambridge, United Kingdom and New York: Cambridge University Press, 881 p.

IPCC. (2006) Guidelines for National Greenhouse Gas Inventories. Japan. IGES. p. 6.24-6.

ITOKAWA, H.; HANAKI, K.; MATSUO, T. (2001) Nitrous oxide production in high-loading biological nitrogen removal process under low COD/N ratio condition. Water Research, v. 35, n. 3, p. 657-64.

KAMPSCHREUR, M.J.; TAN, N.C.; KLEEREBEZEM, R.; PICIOREANU, C.; JETTEN, M.S; VAN LOOSDRECHT, M.C. (2008) Effect of dynamic process conditions on nitrogen oxides emission from a nitrifying culture. Environmental Science \& Technology, v. 42, n. 2, p. 429-35

KAMPSCHREUR, M.J.; TEMMINK, H.; KLEEREBEZEM, R.; JETTEN, M.S.; LOOSDRECHT, M.C. (2009) Nitrous oxide emission during wastewater treatment. Water Research, v. 43, n. 17, p. 4093-103.

KARGI, F.; PAMUKOGLU, M.Y. (2003) Simultaneous adsorption and biological treatment of pre-treated landfill leachate by fed-batch operation. Process Biochemistry, v. 38, n. 10, p. 1413-20.

KLOTZ, M.G.; STEIN, L.Y. (2008) Nitrifier genomics and evolution of the nitrogen cycle. FEMS Microbiology Letters, v. 278, n. 2, p. 146-56.

LEMAIRE, R.; MEYER, R.; TASKE, A.; CROCETTI, G.R.; KELLER, J.; YUAN, $Z$. (2006) Identifying causes for $\mathrm{N}_{2} \mathrm{O}$ accumulation in a lab-scale sequencing batch reactor performing simultaneous nitrification, denitrification and phosphorus removal. Journal of Biotechnology, v. 122, n. 1, p. 62-72.

MARTIN, D.; SALMINEN, J.M.; NIEMI, R.M.; HEISKANEN, I.M.; VALVE, M.J.; HELLSTÉN, P.P.; NYSTÉN, T.H. (2009) Acetate and ethanol as potential enhancers of low temperature denitrification in soil contaminated by fur farms: a pilot-scale study. Journal of Hazardous Materials, v. 163, n. 2-3, p. 1230-8.

MORLEY, N.; BAGGS, E.M. (2010) Carbon and oxygen controls on $\mathrm{N}_{2} \mathrm{O}$ and $\mathrm{N}_{2}$ production during nitrate reduction. Soil Biology \& Biochemistry, v. 42, n. 10, p. $1864-71$

RIVETT, M.O.; BUSS, S.R.; MORGAN, P.; SMITH, J.W.; BEMMENT, C.D. (2008) Nitrate attenuation in groundwater: a review of biogeochemical controlling processes. Water Research, v. 42, n. 16, p. 4215-32.

SCHALK-OTTE, S.; SEVIOUR, R.J.; KUENEN, J.G.; JETTEN, M.S.M. (2000) Nitrous oxide $\left(\mathrm{N}_{2} \mathrm{O}\right)$ production by Alcaligenes Faecalis during feast and famine regimes. Water Research, v. 34, n. 7, p. 2080-8.
SHAW, L.J.; NICOL, G.W.; SMITH, Z.; FEAR, J.; PROSSER, J.I.; BAGGS E.M. (2006) Nitrosospira spp. can produce nitrous oxide via a nitrifier denitrification pathway. Environmental Microbiology, v. 8, n. 2, p. 214-22.

SPANNING, R.; RICHARDSON, D.J.; FERGUSON, S.J. Introduction to the Biochemistry and Molecular Biology of Denitrification. (2007) In: Bothe, H.; Ferguson, S; Newton, W.E. Biology of the Nitrogen Cycle. Amsterdam: Elsevier, p. 198-217.

TALLEC, G.; GARNIER, J.; BILLEN, G.; GOUSAILLES, M. (2008) Nitrous oxide emissions from denitrifying activated sludge of urban wastewater treatment plants, under anoxia and low oxygenation. Bioresource Technology, v. 99, n. 7, p. 2200-9.

TALLEC, G.; GARNIER, J.; BILLEN, G.; GOUSAILLES, M. (2006) Nitrous oxide emissions from secondary activated sludge in nitrifying conditions of urban wastewater treatment plants: effect of oxygenation level. Water Research, v. 40, n. 15, p. 2972-80.

THÖRN, M.; SÖRENSSON, F. (1996) Variation of nitrous oxide formation in the denitrification basin in a wastewater treatment plant with nitrogen removal. Water Research, v. 30, n. 6, p. 1543-7.

TSUNEDA, S.; MIKAMI, M.; KIMOCHI, Y.; HIRATA, A. (2005) Effect of salinity on nitrous oxide emission in the biological nitrogen removal process for industrial wastewater. Journal of Hazardous Materials, v. 119, n. 1-3, p. 93-8

VAN NIEL, E.W.J.; ARTS, P.A.M.; WESSELINK, B.J.; ROBERTSON, L.A.; KUENEN, J.G. (1993) Competition between heterotrophic and autotrophic nitrifiers for ammonia in chemostat cultures. FEMS Microbiology Letters, v. 102, n. 2, p. 109-18.

WICHT, H. (1996) A model for predicting nitrous oxide production during denitrification in activated sludge. Water Science and Technology, v. 34 n. 4-5, p. 99-106.

YE R.W.; THOMAS S.M. (2001) Microbial nitrogen cycles: physiology, genomics and applications. Current Opinion in Microbiology, v. 4, n. 3, p. 307-12

YU, R.; KAMPSCHREUR, M.J.; VAN LOOSDRECHT, M.C.; CHANDRAN, K. (2010) Mechanisms and specific directionality of autotrophic nitrous oxide and nitric oxide generation during transient anoxia. Environmental Science \& Technology, v. 44, n. 4, p. 1313-9.

ZHENG, H.; HANAKI, K.; MATSUO, T. (1994) Production of nitrous oxide gas during nitrification of wasterwater. Water Science and Technology, $v$. 30, n. 6, p. 133-41. 